\title{
Amino Acid Residues that Control pH Modulation of Transport- Associated Current in Mammalian Serotonin Transporters
}

\author{
Yongwei Cao, Ming Li, Sela Mager, and Henry A. Lester \\ Division of Biology, California Institute of Technology, Pasadena, California 91125
}

The rat and human serotonin transporters (rSERT and hSERT, respectively) were expressed in Xenopus oocytes and studied using site-directed mutagenesis, electrophysiological recordings, and $\left[{ }^{3} \mathrm{H}\right] 5-\mathrm{HT}$ uptake measurements. rSERT, but not hSERT, displayed increased transport-associated current at low $\mathrm{pH}$. Chimeras and point mutations showed that, of the 52 nonidentical residues, a single residue at position 490 (threonine in rSERT and lysine in hSERT) governs this difference. Furthermore, potentiation required the glutamate residue at position 493. Cysteine substitution and alkylation experiments showed that residue 493 is extracellular. Cysteine at 493 increased, whereas aspartate decreased, the net charge move-

The mammalian 5-HT transporter (SERT) plays an important role in terminating serotonergic transmission throughout the CNS and in peripheral tissues (Amara and Kuhar, 1993), constitutes a target for antidepressants and drugs of abuse (Rudnick and Clark, 1993), and displays variations in expression that are correlated with personality (Lesch et al., 1996) and with prevalence of affective disorders (Collier et al., 1996). cDNA cloning data show that vertebrate (Blakely et al., 1991; Hoffman et al., 1991; Lesch et al., 1993; Ramamoorthy et al., 1993) and invertebrate (Corey et al., 1994; Demchyshyn et al., 1994) SERT proteins belong to a family of related $\mathrm{Na}^{+}, \mathrm{Cl}^{-}$-coupled neurotransmitter transporters that display overall similarities in the function as well as individual differences in the nature and stoichiometry of cotransported ions, rate of transport, and associated electrophysiological properties (Lester et al., 1994, 1996).

Recent experiments have uncovered four distinct conducting states of rSERT (Mager et al., 1994; Cao et al., 1997). (1) Classical stoichiometric data for SERT predict no net charge movement for each 5-HT molecule (see Fig. 9) (Rudnick and Clark, 1993). Yet electrophysiological recordings from oocytes expressing rSERT revealed current associated with 5-HT uptake (Mager et al., 1994). Previous studies showed that acidic $\mathrm{pH}$ increases this transport-associated current in $\mathrm{rSERT}$; the $\mathrm{EC}_{50}$ for this effect is $7.8 \mu \mathrm{M} \mathrm{H}^{+}, \mathrm{pH}$ 5.1, and the Hill coefficient is 1.1,

\footnotetext{
Received June 23, 1998; accepted July 14, 1998.

This work was supported by Grant DA-09121 from the National Institute on Drug Abuse and Grant NS-11756 from the National Institute of Neurological Disorders and Stroke, by a National Institutes of Health National Research Service Award to Y.C., and by a National Alliance for Research on Schizophrenia and Depression fellowship to S.M. We thank B. J. Hoffman and G. Rudnick for providing cDNAs.

Correspondence should be addressed to Dr. Henry A. Lester, Division of Biology 156-29, California Institute of Technology, Pasadena, CA 91125.

Dr. Cao's present address: Monsanto Company, Mail Code CV-BRG, St. Louis, MO 63198;

Dr. Mager's present address: Department of Physiology, University of North Carolina at Chapel Hill, Chapel Hill, NC 27599-7545.

Copyright (C) 1998 Society for Neuroscience $\quad 0270-6474 / 98 / 187739-11 \$ 05.00 / 0$
}

ment per transported 5-HT molecule. The mutations at this region did not significantly affect other aspects of SERT function, including agonist-independent leakage current, voltagedependent transient current, and $\mathrm{H}^{+}$current. This region may therefore be part of an external gate required for rSERT function. The data and analyses show that, in the absence of detailed structural information, a gate-lumen-gate scheme is useful for interpreting results from mutations that alter functional properties of neurotransmitter transporters.

Key words: 5-HT; Xenopus oocyte; protons; channels; electrophysiology; sodium

suggesting the involvement of a single acidic side chain. However, $\mathrm{pH}$ has no effect on 5-HT uptake (except at extreme $\mathrm{pH}$ ), indicating that $\mathrm{pH}$ may affect this conducting state without affecting 5-HT binding and transport (Cao et al., 1997). Several other neurotransmitter transporters also display "excess" transportassociated current (Lester et al., 1996; Sonders and Amara, 1996; Galli et al., 1997). (2) There is a $\mathrm{Na}^{+}$leakage current in the absence of 5-HT (Mager et al., 1994). High-resolution patch recordings revealed that the transport-associated current and the leakage current arise from unitary events that resemble singlechannel openings, but the single-channel events that underlie the transport-associated current occur at a frequency of only one per $\sim 750$ transported 5-HT molecules (Lin et al., 1996). (3) There is a voltage-dependent transient current (Mager et al., 1994). (4) There is an additional current at low $\mathrm{pH}$, probably carried by protons (Cao et al., 1997).

In this study, we have sought and found mutations that modify the first of these currents and its relationship to 5-HT transport. We find that the $\mathrm{pH}$ dependence of the transport-associated current differs between human (hSERT) and rat (rSERT) transporters. Previous experiments have successfully exploited chimeras between homologous transporters within the $\mathrm{Na}, \mathrm{Cl}$ superfamily that specifically transport different substrates (Buck and Amara, 1994, 1995; Giros et al., 1994), and chimeras between the orthologous hSERT and rSERT transporters were used to localize a region distal to amino acid 532 that governs species preferences for the binding of imipramine and D-amphetamine (Barker et al., 1994). We have also exploited hSERT-rSERT chimeras. Analysis of point mutations then led to the conclusion that the set of residues governing this difference lies in an extracellular portion of the protein and may participate in an external gating process of the transporter.

\section{MATERIALS AND METHODS}

Plasmid DNAs, site-directed mutagenesis, and mRNA synthesis. The rSERT cDNA (Hoffman et al., 1991) was used in the expression vector 

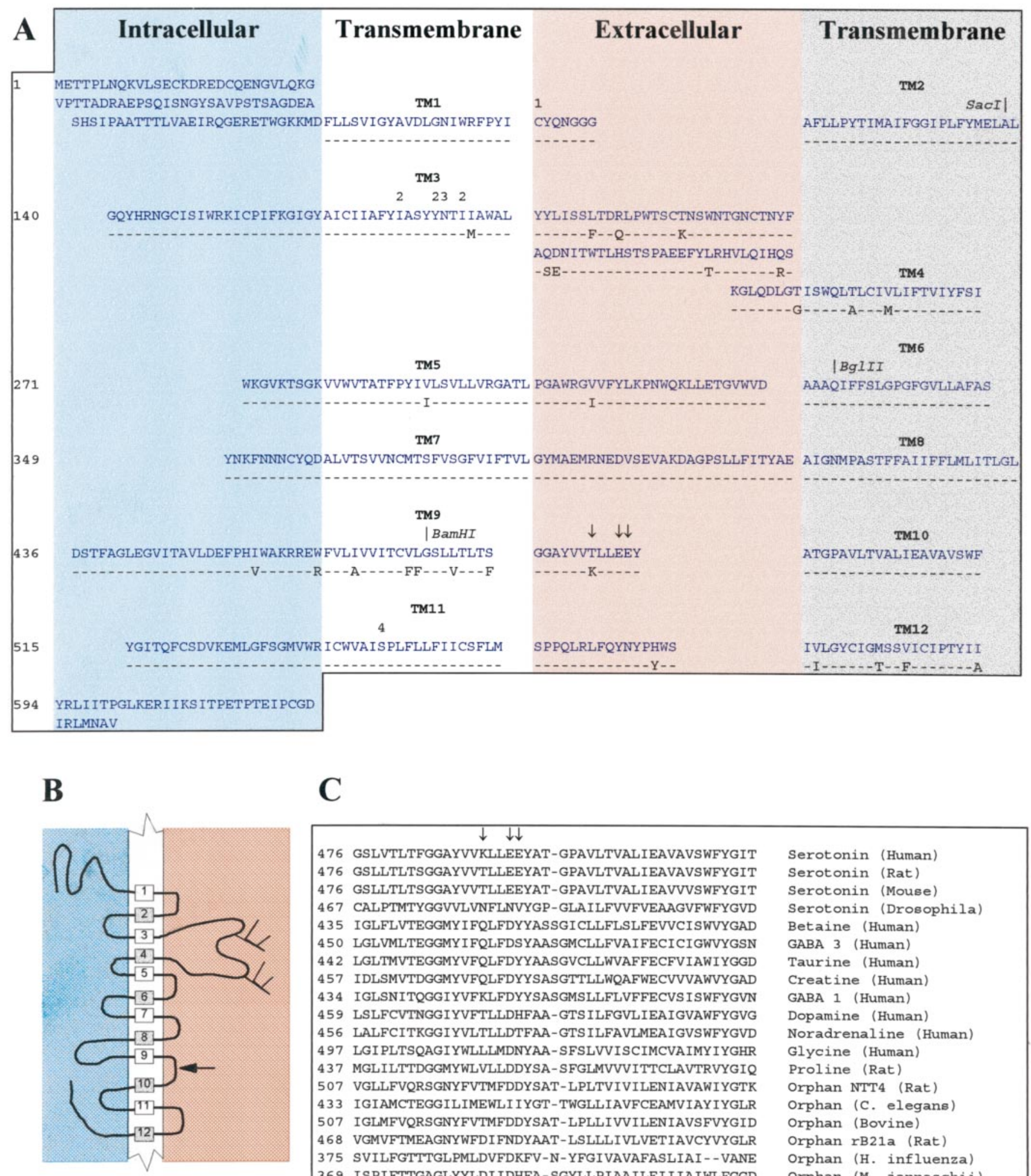

C

\begin{tabular}{|c|c|c|}
\hline & $\downarrow \downarrow$ & \\
\hline 476 & GSLVTLTFGGAYVVKLLEEYAT-GPAVLTVALIEAVAVSWFYGIT & Serotonin (Human) \\
\hline 476 & GSLLTLTSGGAYVVTLLEEYAT-GPAVLTVALIEAVAVSWFYGIT & Serotonin (Rat) \\
\hline 476 & GSLLTLTSGGAYVVTLLEEYAT-GPAVLTVALIEAVVVSWFYGIT & Serotonin (Mouse) \\
\hline 467 & CALPTMTYGGVVLVNFLNVYGP-GLAILFVVFVEAAGVFWFYGVD & Serotonin (Drosophila) \\
\hline 435 & IGLFLVTEGGMY IFQLFDYYASSGI CLLFLSLFEVVCISWVYGAD & Betaine (Human) \\
\hline 450 & LGLVMLTEGGMY IFQLFDSYAASGMCLLFVAIFECICIGWVYGSN & GABA 3 (Human) \\
\hline 442 & LGLTMVTEGGMYVFQLFDYYAASGVCLLWVAFFECFVIAWIYGGD & Taurine (Human) \\
\hline 457 & IDLSMVTDGGMYVFQLFDYYSASGTTLLWQAFWECVVVAWVYGAD & Creatine (Human) \\
\hline 434 & IGLSNITQGGIYVFKLFDYYSASGMSLLFLVFFECVS ISWFYGVN & GABA 1 (Human) \\
\hline 459 & LSLFCVTNGGIYVFTLLDHFAA-GTS ILFGVLIEAIGVAWFYGVG & Dopamine (Human) \\
\hline 456 & LALFCITKGGIYVLTLLDTFAA-GTS ILFAVLMEAIGVSWFYGVD & Noradrenaline (Human) \\
\hline 497 & LGI PLTSQAGIYWLLLMDNYAA-SFSLVVISCIMCVAIMY IYGHR & Glycine (Human) \\
\hline 437 & MGLILTTDGGMYWLVLLDDYSA-SFGLMVVVITTCLAVTRVYGIQ & Proline (Rat) \\
\hline 507 & VGLLFVQRSGNYFVTMFDDYSAT-LPLTVIVILENIAVAWIYGTK & Orphan NTT4 (Rat) \\
\hline 433 & IGIAMCTEGGILIMEWLI IYGT - TWGLLIAVFCEAMVIAY I YGLR & Orphan (C. elegans) \\
\hline 507 & IGLMFVQRSGNYFVTMFDDYSAT-LPLLIVVILENIAVSFVYGID & Orphan (Bovine) \\
\hline 468 & VGMVFTMEAGNYWFDIFNDYAAT-LSLLLIVLVETIAVCYVYGLR & Orphan rB21a (Rat) \\
\hline 375 & SVILFGTTTGLPMLDVFDKFV-N-YFGIVAVAFASLIAI - -VANE & Orphan (H. influenza) \\
\hline 369 & ISPIFTTGAGLYYLDI IDHFA-SGYLLPIAAILEI I IAIWLFGGD & Orphan (M. jannaschii) \\
\hline
\end{tabular}

Figure 1. Annotated amino acid sequences for rSERT and related transporters. A, Alignment between rSERT and hSERT (GenBank accession numbers X63253 and L05568, respectively). Putative membrane topology is indicated by the blue (intracellular), white (transmembrane, N terminal near the intracellular face), pink (extracellular), and gray (transmembrane, $\mathrm{N}$ terminal near the extracellular face) regions. Residues in rSERT are shown in dark blue letters; residues in hSERT (shown below rSERT in black) are dashed where identical to those in rSERT and shown explicitly where the two sequences differ, but for simplicity, differences are not shown for the $\mathrm{N}$ - and $\mathrm{C}$-terminal intracellular regions (22 and 4 differences, respectively). Positions of conserved restriction enzyme sites that were used for chimera construction are marked by the enzyme name. Residues in which mutations have been studied in the present experiments are marked by $\downarrow$. Numbers mark the positions in which mutations were characterized (Figure legend continues) 
pAM V-PA (Nowak et al., 1998) as described (Lin et al., 1996). The hSERT cDNA was isolated by Dr. Beth J. Hoffman (National Institute of Mental Health). The rSERT cDNA fragment was radiolabeled by random priming and used to isolate a $4.5 \mathrm{~kb} \mathrm{cDNA}$ clone corresponding to a partial cDNA of the hSERT from a human raphe cDNA library (B. J. Hoffman, personal communication) constructed in pCDSP6T7 (Usdin et al., 1991). The $5^{\prime}$ end of the coding region was isolated (B. J. Hoffman, personal communication) by rapid amplification of cDNA ends (Frohman et al., 1988) from human raphe poly $\left(\mathrm{A}^{+}\right)$RNA followed by PCR using two gene-specific 22-nt oligomers [upstream (sense), $5^{\prime}$-TTG GGA TCC TTG GCA GAT GGA C-3'; downstream (antisense), 5'GCT GGT CCA GGG CAG CTG GTC C-3']. A complete cDNA was constructed by ligation of a Bam HI/Sst digest of this PCR product to an Sst $\mathrm{I} / E \mathrm{E} o \mathrm{RV}$ digest of the $3^{\prime}$ end of the cDNA in pSP73 (New England Biolabs, Beverly, MA; http://www.neb.com). The sequence of the complete cDNA was verified by double-stranded sequencing. The cDNA insert was excised by digestion with $S m a \mathrm{I}$ and $\mathrm{XbaI}$ and transferred into the EcoRV and $X b a \mathrm{I}$ sites of a modified pAMV-PA vector (the AMV sequence was removed).

The C109A rSERT mutant was provided by Dr. Gary Rudnick (Yale University School of Medicine) (Chen et al., 1997a) and was also transferred into the pAMV-PA vector. Site-directed mutagenesis was performed using a PCR method (Higuchi, 1990). PCR products were subcloned into PAMV-PA, and the presence of the intended mutation was confirmed by sequencing. Plasmid DNAs were prepared using the Qiagen (Hilden, Germany; http://www.qiagen.com) miniprep kit and were linearized by NotI digestion. RNA was synthesized in vitro using the Ambion (Austin, TX; http://www.ambion.com) transcription kit.

Sequence alignments were performed using LaserGene software (DNASTAR, Madison, WI; http://www.dnastar.com). For the alignments of the short stretch (see Fig. 1C), a total of $65 \mathrm{Na}^{+}, \mathrm{Cl}^{-}$dependent neurotransmitter transporters and orphan transporters was selected from the GenBank database. A candidate sequence (the human sequence when available) was then selected from each cluster and was realigned.

Oocyte expression. Stage V and VI oocytes were isolated and injected with $\sim 20 \mathrm{ng}$ of mRNA in 30-50 $\mathrm{nl}$ of water. Injected oocytes were then incubated 3-7 d at $19^{\circ} \mathrm{C}$ for translation (Quick and Lester, 1994).

Electrophysiology. Voltage-clamp experiments were performed using the two-electrode technique (Mager et al., 1994). Data acquisition and analysis were performed using the pCLAMP programs (Axon Instruments, Foster City, CA; http://www.axonet.com). Normal Na ${ }^{+}$Ringer's solution contained $100 \mathrm{~mm} \mathrm{NaCl}, 2 \mathrm{~mm} \mathrm{KCl}, 1 \mathrm{~mm} \mathrm{MgCl}_{2}, 5 \mathrm{~mm}$ HEPES, and $5 \mathrm{~mm}$ MES. Solution $\mathrm{pH}$ was adjusted with $\mathrm{NaOH}$ or $\mathrm{HCl}$ to obtain $\mathrm{pH} 7.4$ or 5.5 as indicated in the text. For cation substitution experiments, $\mathrm{NaCl}$ was replaced by equimolar $\mathrm{N}$-methyl-D-glucamine (NMDG) chloride. Solution changes were made with electrically operated valves (Auto-Mate Scientific, San Francisco, CA). All recordings were performed at room temperature $\left(21-22^{\circ} \mathrm{C}\right)$. Tracings in the figures represent similar traces recorded from at least three oocytes in each of two or three batches. A Fourier transform algorithm was used to suppress $60 \mathrm{~Hz}$ interference in some traces (see Fig. 3).

Sulfhydryl modification by methanethiosulfonate derivatives. Methanethiosulfonate-ethylammonium (MTSEA) and MTS-ethylsulfonate (MTSES) (Toronto Research Chemical Company, Toronto, Canada) were used for irreversible, covalent modification of cysteine residues. After an initial electrophysiological recording, oocytes were removed from the voltageclamp chamber and incubated in $\mathrm{Na}^{+}$Ringer's solution containing 0.5 mM MTSEA or $2 \mathrm{~mm}$ MTSES for $10 \mathrm{~min}$. After incubation, the oocytes were washed and voltage-clamped a second time; these recordings were compared with the preincubation records to assess the effects of sulfhydryl modification.

MTS compounds were prepared as $1.0 \mathrm{M}$ solutions in $\mathrm{H}_{2} \mathrm{O}$ and kept at $-80^{\circ} \mathrm{C}$. These stock solutions were diluted in the appropriate buffer solution at room temperature just before exposure to the oocyte.

5-HT uptake. 5-HT uptake was usually measured by a 3 min incubation

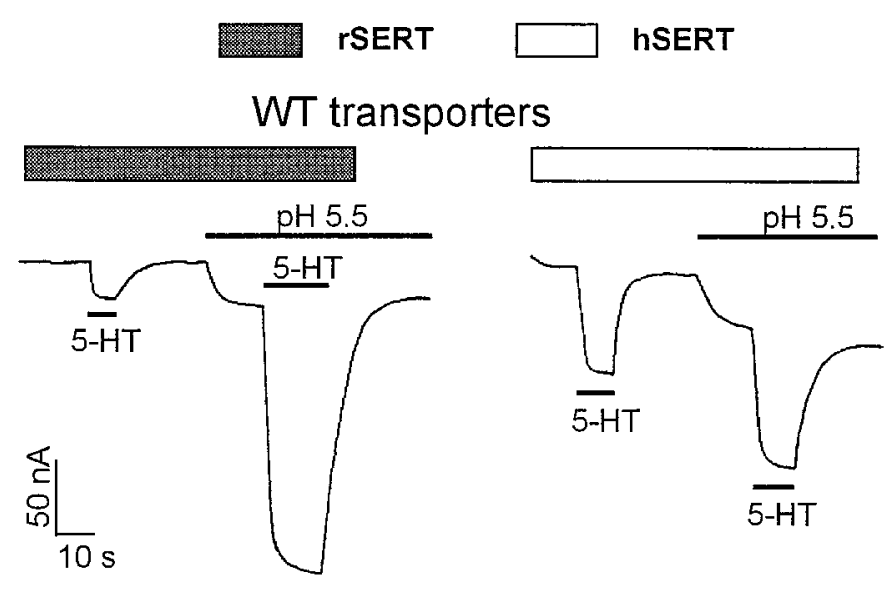

Figure 2. Effects of acidic $\mathrm{pH}$ on transport-associated current in wildtype (WT) rSERT and hSERT. Note that $\mathrm{pH} 5.5$ solution produces an additional inward current in the absence of 5-HT in both transporters (Cao et al., 1997). However, only in rSERT the pH 5.5 solution also increases the transport-associated current during 5-HT application. The holding potential was $-40 \mathrm{mV}$. The base solution was normal $\mathrm{Na}^{+}$ Ringer's solution, $\mathrm{pH}$ 7.4. Applications of acidic $\mathrm{Na}^{+}$Ringer's solution, $\mathrm{pH} 5.5$, and 5-HT (3 $\mu \mathrm{M})$ are indicated by horizontal bars.

in $300 \mu \mathrm{l}$ of uptake solution containing $1 \mu \mathrm{M}\left[{ }^{3} \mathrm{H}\right] 5-\mathrm{HT}$. Oocytes were washed once with the uptake solution before uptake began and three times after uptake ended. Oocytes were then solubilized in $2 \%$ SDS. The $\left[{ }^{3} \mathrm{H}\right] 5-\mathrm{HT}$ uptake was determined by liquid scintillation counting.

To determine 5-HT uptake during voltage clamp, we placed the oocyte in the recording chamber and held the membrane potential at $-40 \mathrm{mV}$. The solution flow was then stopped, and $\left[{ }^{3} \mathrm{H}\right] 5-\mathrm{HT}$ was added with gentle stirring to a final concentration of $1 \mu \mathrm{M}$. Current induced by 5-HT was simultaneously recorded on a chart recorder. After $1 \mathrm{~min}$, the recording chamber was perfused with Ringer's solution for $1 \mathrm{~min}$.

\section{RESULTS}

Figure 1, $A$ and $B$, presents the primary amino acid sequence and putative membrane topology of rSERT, which was used for most of our experiments, and of the human homolog hSERT (Blakely et al., 1991; Hoffman et al., 1991; Lesch et al., 1993; Ramamoorthy et al., 1993). Regions of special interest are marked. Sequence comparisons between rSERT and hSERT show that the two proteins have a high degree of similarity, with 52 different residues spanning a total of 630 amino acids (Fig. 1A).

\section{Acidic pH potentiates transport-associated current markedly in rSERT but not in hSERT}

We reported previously that acidic $\mathrm{pH}$ significantly increased the transport-associated current in oocytes expressing rSERT (Cao et al., 1997). Figure 2 recapitulates this finding. In these experiments (see also the experiments described in Figs. 4-6 below), the $\mathrm{pH}$-dependent enhancement of the transport-associated current was analyzed by comparing the transport-induced currents in individual oocytes at $\mathrm{pH} 7.4$ with those at $\mathrm{pH}$ 5.5. This tactic presumably eliminates distortions from variations in expression level among the various constructs. In contrast, for hSERT ex-

\section{$\leftarrow$}

in previous studies: 1, C109 (Chen et al., 1997a); 2, I172, Y176, and I179 (Chen et al., 1997b); 3, N177 (Lin et al., 1996); and 4, S545 (Sur et al., 1997). The C109A mutant was used for some of the experiments in this paper (see Figs. 6, 7). TM, Transmembrane. B, Summary of the membrane topology shown in $A$. $C$, Alignments for 19 transporters, including $\sim 44$ residues in the putative extracellular region studied in this paper, flanking the E493 region of rSERT. Initially, a total of $65 \mathrm{Na}^{+}, \mathrm{Cl}^{-}$-dependent neurotransmitter transporters and orphan transporters was selected from the GenBank database. A candidate sequence (the human sequence if possible) was then selected from each cluster and was realigned. The rSERT residues mutated in this study are shown by $\downarrow$. 

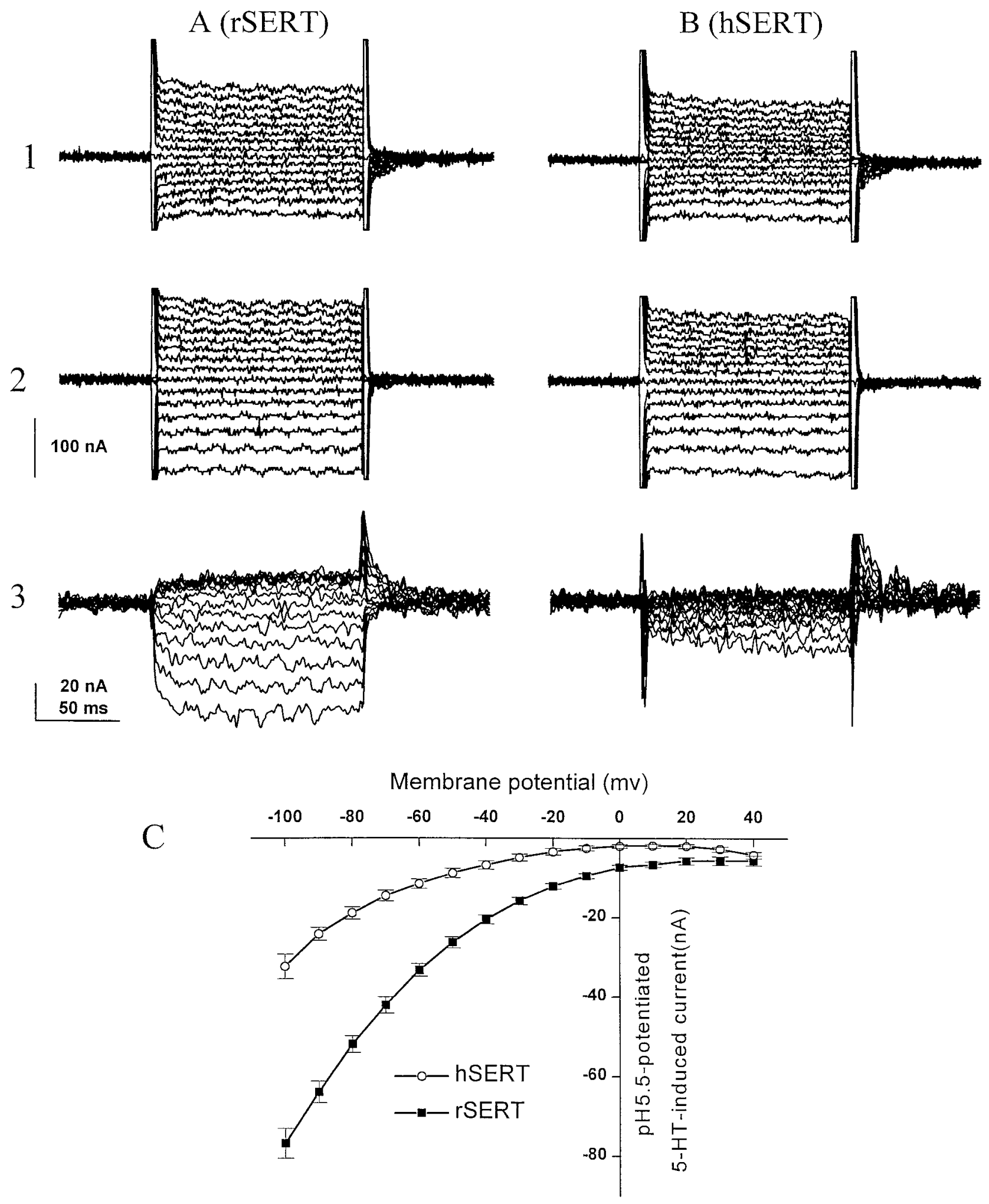

Figure 3. Voltage dependence of the transport-associated current at pH 5.5. A, Data for a representative oocyte expressing rSERT. Row 1, Voltage-clamp traces in the absence of 5-HT, $\mathrm{pH} 5.5$. The membrane potential was held at $-40 \mathrm{mV}$ and jumped to test potentials at $10 \mathrm{mV}$ increments between $+40 \mathrm{mV}$ and $-100 \mathrm{mV}$ for $100 \mathrm{msec}$. Row 2, Traces for the same voltage-clamp protocol applied 3 min later in the presence of 5-HT (3 $\mu \mathrm{M}$ ), $\mathrm{pH}$ 5.5. Row 3, Subtraction revealing the 5-HT-induced current at $\mathrm{pH}$ 5.5. Note that the subtracted traces are displayed at a higher gain. $B$, Data for a representative oocyte expressing hSERT, with voltage-clamp protocols and subtraction described in $A$. The oscillations in the final epoch of row 3 are an artifact of the algorithm that suppressed $60 \mathrm{~Hz}$ interference earlier in the traces. $C$, The transport-associated current at pH 5.5 plotted versus membrane potential. The current was averaged over the final $30 \mathrm{msec}$ of the test pulse. Data are mean \pm SEM for five rSERT and four hSERT oocytes. 
pressed in oocytes, the transport-associated current was increased only slightly (20\% in the trace in Fig. 2, right) and actually decreased in other experiments. For the batch of oocytes that yielded our most complete set of comparative data, transportassociated current for rSERT and hSERT at $-40 \mathrm{mV}$ increased to $399 \pm 33 \%$ and decreased to $63 \pm 1 \%$ (mean $\pm \mathrm{SEM}$; five oocytes), respectively, of the value at $\mathrm{pH} 7.4$ when external $\mathrm{pH}$ was lowered to 5.5.

Most measurements were made at a holding potential of -40 $\mathrm{mV}$ for consistency with our previous study (Cao et al., 1997), but in Figure 3, we analyze the voltage dependence of the transportassociated current at low pH. For both rSERT and hSERT, the transport-associated current at pH 5.5 increased at more negative potentials and displayed no clear reversal at positive potentials. This current-voltage relation is similar to that for the measurements at pH 7.4 (Mager et al., 1994), but the larger size of the current at $\mathrm{pH} 5.5$ for rSERT allowed more accurate measurements. (We note that oocytes expressing hSERT generally displayed larger transport-associated currents than did those expressing rSERT, so that the enhanced current for rSERT was only twice as large as that for hSERT, even though the fractional enhancement at pH 5.5 was approximately four times larger for rSERT than for hSERT. We have not systematically explored the basis for the differing transport-associated currents, but the difference has persisted with two series of mRNA syntheses and with three oocyte batches.) At all membrane potentials between -20 and $-100 \mathrm{mV}, \mathrm{pH} 5.5$ enhanced the transport-induced current for rSERT several fold, but there was little enhancement or a decrease for hSERT. Other electrophysiological properties of hSERT, such as the presence of leakage current, $\mathrm{H}^{+}$current, and transient current, did not differ significantly from those of rSERT (data not shown). Thus, this difference in the fractional enhancement of the transport-associated current by low $\mathrm{pH}$ constitutes a specific and limited electrophysiological distinction between rSERT and hSERT.

\section{The different residues at position 490 are responsible for the different $\mathrm{pH}$ sensitivities of rSERT and hSERT}

We constructed chimeric rSERT-hSERT proteins by swapping corresponding cDNA segments generated by restriction enzyme digestion at several conserved sites ( $\mathrm{SacI}, \mathrm{Bgl \textrm {II }}$, and $\mathrm{BamH \textrm {H }}$; see Fig. 1A). Measurements on oocytes expressing these chimeric transporters showed that transport-associated current was present in all four chimeras tested, but marked potentiation by $\mathrm{pH} 5.5$ was seen only in chimeras containing the C-terminal 155 residues or more of the rSERT sequence (Fig. 4). Evidently this electrophysiological difference can be localized to a part of the transporter molecule consisting of the $\mathrm{C}$-terminal 155 residues.

We next asked whether the electrophysiological difference can be localized to a specific residue. In these 155 positions at the $\mathrm{C}$ terminal, rSERT and hSERT differ by 12 amino acid residues (Fig. 1 $A$; four differences are not shown explicitly in the intracellular $\mathrm{C}$ terminal). We therefore began to mutate these residues individually. We first chose residues at positions 483 (rS483F; typical trace shown in Fig. 5A), 490 (rT490K; Fig. 5B), and 572 (rH572Y; Fig. 5C), because these residues are (1) polar and (2) thought to be in extracellular or transmembrane portions of the protein and thus more likely to respond to extracellular $\mathrm{pH}$ changes. Each of the three mutants was functional in oocytes, but only rT490K (Fig. 5B) showed a dramatically decreased low-pH potentiation of the transport-associated current. To verify that position 490 governs the difference in $\mathrm{pH}$ sensitivity between

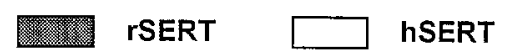

\section{Chimeric transporters}
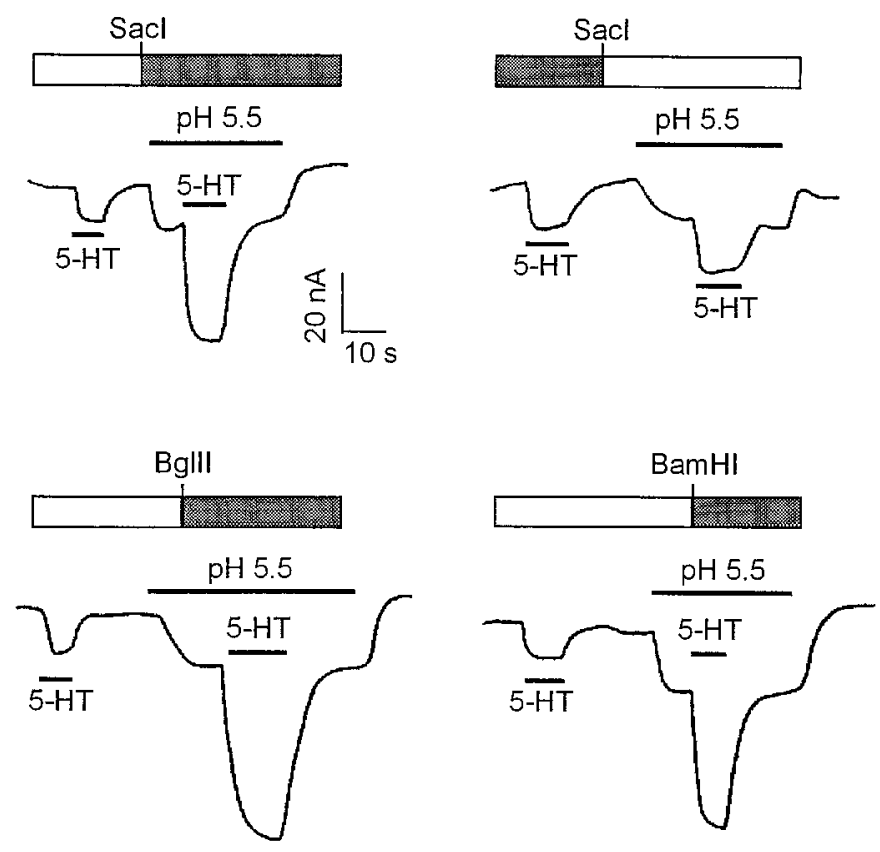

Figure 4. Localization of a region responsible for $\mathrm{pH}$ dependence of the transport-associated current. Experiments similar to those of Figure 2 were performed on chimeric rSERT-hSERT transporters. The constructs used restriction sites for $\mathrm{SacI}, \mathrm{Bgl \textrm {II }}$, and BamHI. For locations of each restriction enzyme site, see Figure 1 . Note that all the data are consistent with the interpretation that $\mathrm{pH} 5.5$ enhances the transport-associated currents in transporters containing C-terminal sequences from rSERT. The BamHI construct contains the fewest rSERT residues (155) and indicates that the sequences governing $\mathrm{pH} 5.5$ enhancement are located in the C-terminal 155 residues.

rSERT and hSERT, we made a reverse mutant in which K490 in hSERT was mutated to threonine (hK490T; Fig. 5D). This reverse mutation conferred an rSERT-like $\mathrm{pH}$ sensitivity on hSERT; the transport-associated current was now significantly increased by acidic $\mathrm{pH}$.

\section{Protonation at E493 is mainly responsible for low-pH potentiation of transport-associated current}

Previous results showed that the $\mathrm{H}^{+}$potentiation of the transport-associated current displays a typical Michaelis-Menten relationship with an $\mathrm{EC}_{50}$ of $7.8 \mu \mathrm{M} \mathrm{H} \mathrm{H}^{+}, \mathrm{pH}$ 5.1, and a Hill coefficient of 1.1, indicating that a single amino acid with $\mathrm{p}_{\mathrm{Ka}} \sim$ 5.1 might be responsible for low-pH potentiation (Cao et al., 1997). Threonine at position 490 is unlikely to be a candidate because its free-solution $\mathrm{p}_{\mathrm{Ka}}$ is $\sim 9.1$. However, there are two consecutive glutamate residues $\left(\mathrm{p}_{\mathrm{Ka}} \sim 4.7\right)$ at positions 493 and 494, just downstream of T490 (Fig. 1A). We suspected that protonation of one or both of these glutamate residues might actually be responsible for low-pH potentiation. To test this hypothesis, we mutated E493 and E494 in rSERT to glutamine and expressed the mutated transporters in oocytes. Results showed that a single mutation at 493 only (E493Q) still allowed low-pH potentiation but at a reduced level (approximately twofold vs fourfold for WT rSERT at $\mathrm{pH}$ 5.5; Fig. $5 E$ ). Mutation at 
A. $\mathrm{rS} 483 \mathrm{~F}$

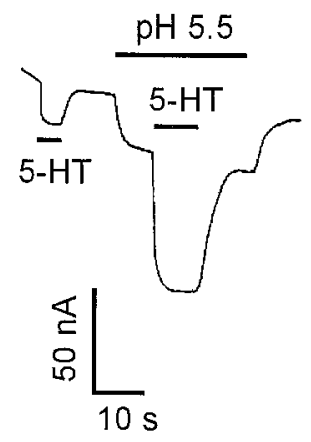

E. E493Q

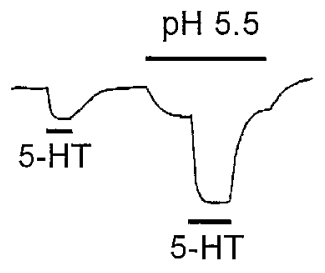

Figure 5. Effects of acidic $\mathrm{pH}$ on transport-associated current from the point mutants $\mathrm{rS} 483 \mathrm{~F}(A), \mathrm{rT} 490 \mathrm{~K}(B)$, rH572Y $(C)$, hK490T $(D)$, E493Q $(E)$, and E494Q $(F)$ and the double point mutant E493Q-E494Q $(G)$. Recording conditions are described in the legend to Figure 2.
Figure 6. Comparisons between transportassociated current in C109A and C109AE493C. $A, B$, Effects of acidic $\mathrm{pH}$ on transport-associated current in typical traces from C109A $(A)$ and C109A-E493C $(B)$. Recording conditions are described in the legend to Figure 2. $C, D$, Effects of cysteine-modifying reagents MTSEA and MTSES on transport-associated current from C109A $(C)$ and C109A-E493C (D). Transport-associated currents were recorded both before and after MTSreagent treatments and were normalized to the current recorded before treatment. Error bars represent the SD in measurements from three oocytes.
B. $\mathrm{rT} 490 \mathrm{~K}$

C. $\mathrm{rH} 572 \mathrm{Y}$

D. hK490T

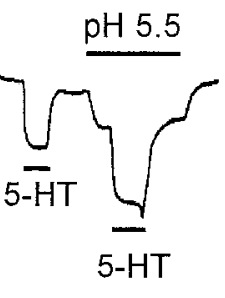

F. E494Q

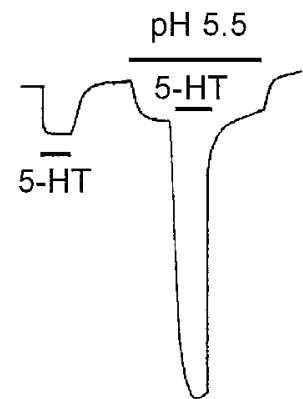

G. E493Q/E494Q

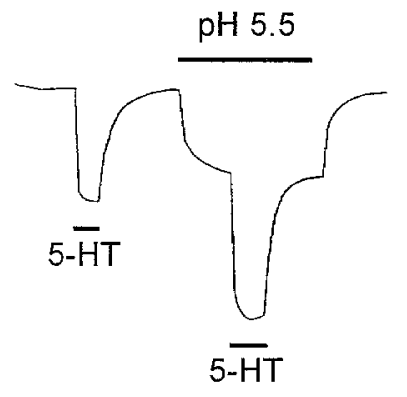

\section{A. C109A}
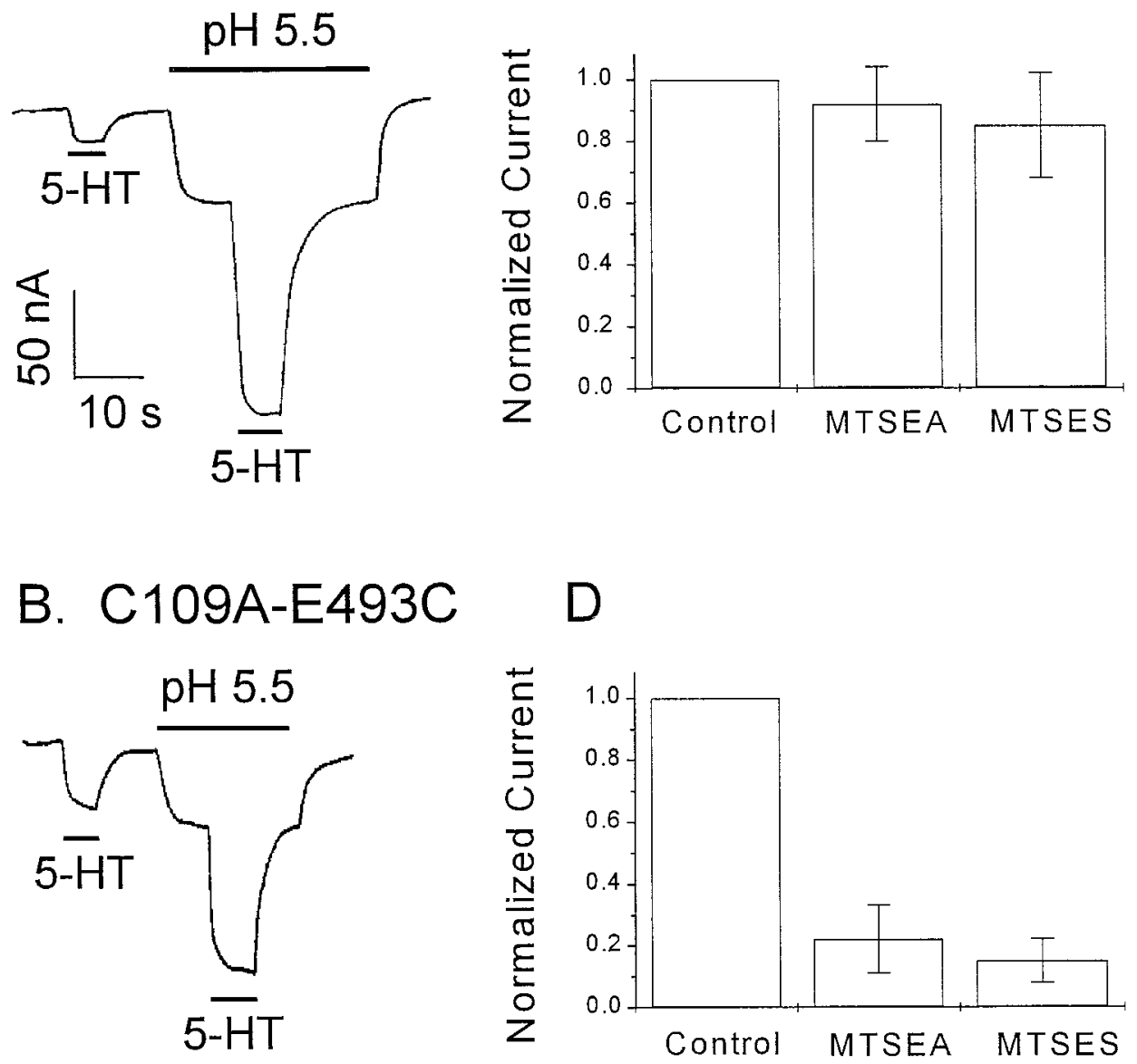

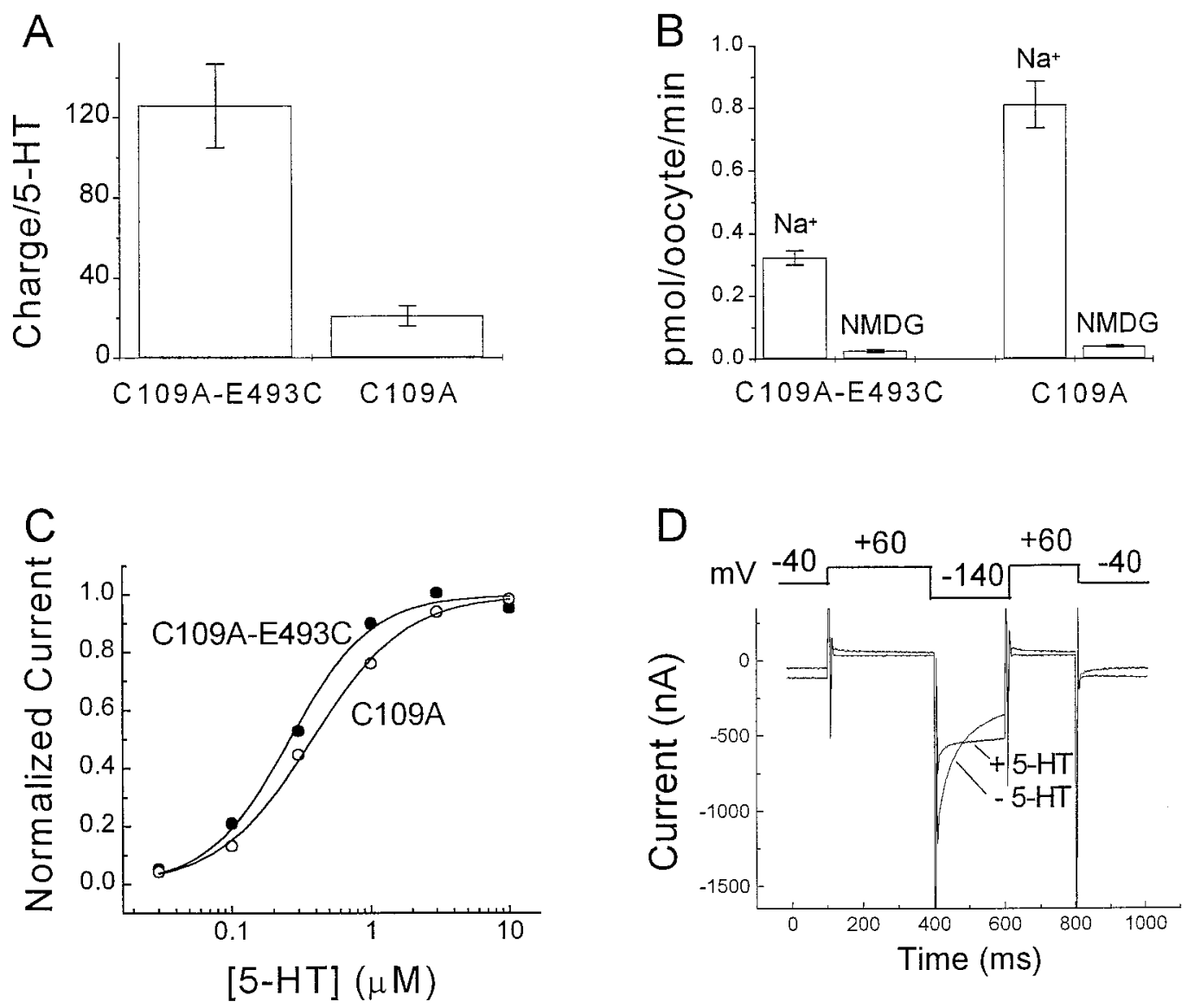

Figure 7. Functional effects of the E493C mutation. A, Comparison of C109A and C109A-E493C on the ratio of net charge movement to net 5-HT uptake. Error bars represent the SD in measurements from three oocytes. B, Comparison of C109A-E493C and a control (C109A) on the Na ${ }^{+}$ dependence of 5-HT uptake. NMDG was used as a substitute for $\mathrm{Na}^{+}$. Error bars represent the SD in measurements from six oocytes. $C$, Dose-response relationship for the transport-associated current. Data were fit by nonlinear regression to the Hill equation. $D$, Voltage-dependent transient current in the C109A-E493C mutant and inhibition of this current by 5-HT. Current traces recorded in the presence $(+)$ and absence $(-)$ of $5-\mathrm{HT}(3 \mu \mathrm{M})$ are superimposed. The voltage protocol is shown at the top.

494 only (E494Q) displayed low-pH potentiation equal to that of WT rSERT (fourfold at pH 5.5; Fig. 5F). However, a double mutation at both 493 and 494 (E493Q-E494Q) almost completely abolished low-pH potentiation (Fig. $5 G$ ). These results suggest that E493 is mainly responsible for the low-pH potentiation. In the absence of E493, E494 partially fills the same role and allows for a moderate level of potentiation. However, potentiation also requires the absence of a positively charged residue at position 490 , as though a lysine-glutamate salt bridge prevents protonation of the glutamate; and hSERT but not rSERT contains the lysine at position 490 .

\section{E493 is exposed to the extracellular solution}

On the basis of standard topology models (Fig. $1 A, B$ ), E493 is located in the extracellular loop between the 9th and the 10th transmembrane segments. To verify the hypothesis of extracellular localization, we mutated E493 to cysteine and tested whether the mutated protein became sensitive to the externally applied cysteine-modifying reagents MTSEA and MTSES. Because WT rSERT is already sensitive to externally applied cysteine reagents, we chose the cysteine reagent-insensitive mutant C109A as our control transporter for this series of experiments (Chen et al., 1997a). Confirming the work of Chen et al. (1997a), we found that this mutant (denoted by the number 1 in Fig. $1 A$ ) functioned similarly to WT rSERT except for its reduced sensitivity to cysteine reagents (Chen et al., 1997a). Like WT rSERT, C109A showed an approximately fourfold increase in transportassociated current at pH 5.5 (Fig. 6A). As expected, incubation of C109A-expressing oocytes in MTSEA $(0.5 \mathrm{~mm})$ or MTSES (2 $\mathrm{mM}$ ) for $10 \mathrm{~min}$ did not change the amplitude of the transportassociated current (Fig. 6C). The E493C mutant constructed on the C109A background (i.e., C109A-E493C double mutant) showed reduced low-pH potentiation of transport-associated current (two- to threefold at $\mathrm{pH} 5.5$ ) (Fig. 6B). This result is expected from the data on the E493Q mutant (Fig. 5E). Importantly, the E493C mutant became sensitive to both MTSEA and MTSES (Fig. 6D). These results suggest that E493 is exposed to the extracellular solution.

\section{E493C increases, and E493D decreases, the net charge movement per 5-HT molecule}

Our previous results showed that acidic $\mathrm{pH}$ (in the range of 5.5 to 6.5 compared with $\mathrm{pH} 7.5$ ) increases transport-associated current but does not change 5-HT uptake (Cao et al., 1997), indicating that $\mathrm{H}^{+}$could increase the ionic flux for each 5-HT molecule further. To test whether this is also the case for the C109AE493C mutant, we performed $\left[{ }^{3} \mathrm{H}\right] 5-\mathrm{HT}$ uptake experiments under voltage-clamp conditions so that $\left[{ }^{3} \mathrm{H}\right] 5-\mathrm{HT}$ uptake and 

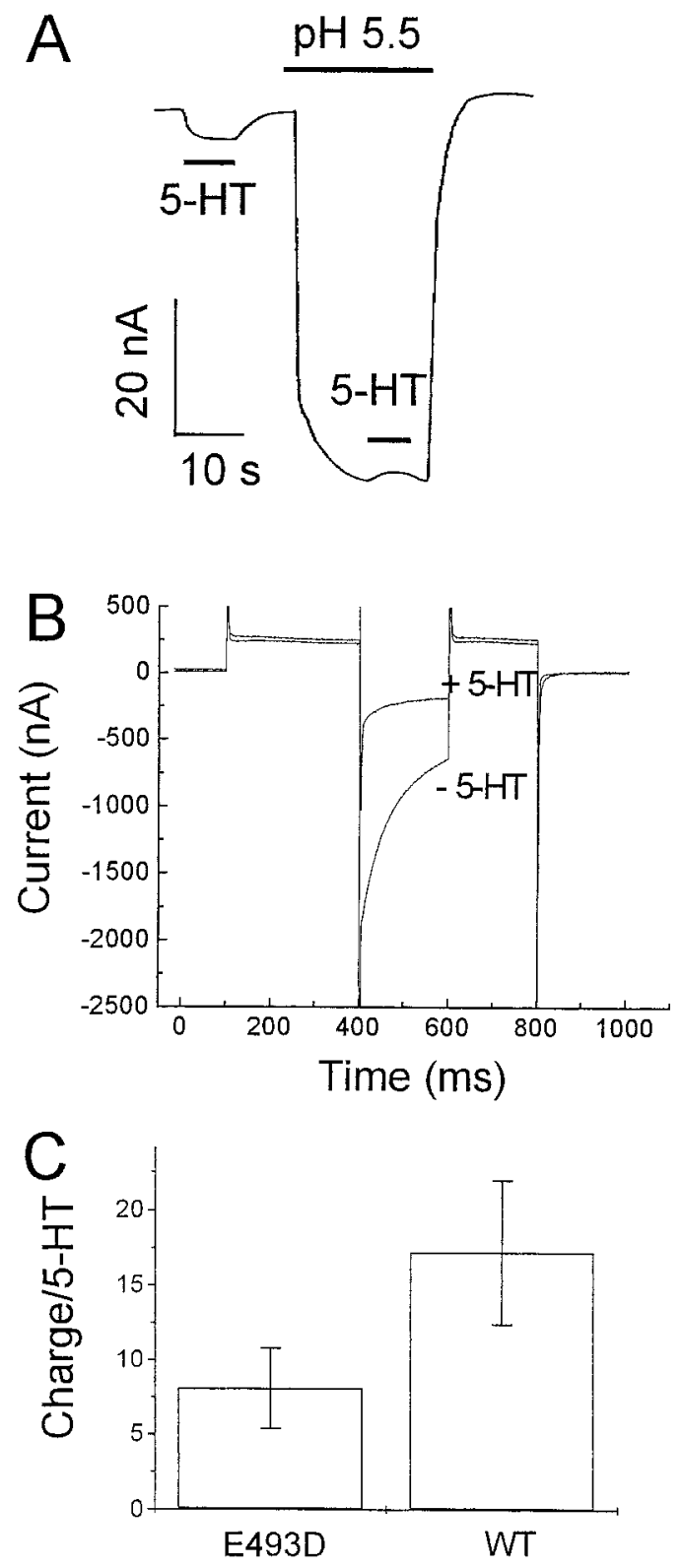

Figure 8. Characterization of the E493D mutant. A, Effect of acidic $\mathrm{pH}$ on transport-associated current. Recording conditions are described in the legend to Figure 2. B, Transient current in E493D and inhibition of this current by 5 -HT. Current traces recorded in the presence and absence of 5-HT (3 $\mu \mathrm{M})$ are superimposed. The voltage protocol is the same as that shown in Figure 7D.C, Comparison of E493D and WT on the ratio of net charge movement to net 5-HT uptake. Error bars represent the SD in measurements from three oocytes.

total ionic flux could be measured simultaneously. We found that the E493C mutant transported 5.5-fold more charges per 5-HT molecule (ratio of $126 \pm 21$ charges per 5 -HT; $n=3$ ) than did the control C109A (21 \pm 5 charges per 5-HT; $n=3)$ (Fig. 7A). Similar results were found with the E493Q mutant (data not shown). The E493C mutation did not change the amplitude of the leakage current or the $\mathrm{Na}^{+}$dependence of 5-HT uptake (Fig. $7 B)$. The transport-associated current depended on [5-HT] with similar $\mathrm{EC}_{50}$ values $(0.3 \pm 0.1$ and $0.4 \pm 0.1 \mu \mathrm{M} ; n=3)$ and Hill coefficients $(1.4 \pm 0.2$ and $1.3 \pm 0.1)$ for C109A-E493C and the control C109A, respectively (Fig. 7C). The $\mathrm{EC}_{50}$ for $\mathrm{Na}^{+}$was not significantly changed (data not shown). The hyperpolarizationinduced transient current found in WT rSERT (Mager et al., 1994) was also present in C109A-E493C, although with reduced amplitude (Fig. 7D). As we expected from the data on WT SERT, this transient current was blocked by 5-HT (Fig. 7D). Thus, the most significant changes produced by the E493C mutation were (1) reduced $\mathrm{pH}$ sensitivity of transport-associated current and (2) increased net charge movement per 5-HT uptake.

Sequence alignment of the known $\mathrm{Na}^{+}, \mathrm{Cl}^{-}$-coupled neurotransmitter transporters and some orphan transporters reveals that the negatively charged residue at position 493 of rSERThSERT is highly conserved, perhaps even in two orphan transporters from procaryotes (Fig. 1C). However, only mammalian 5-HT transporters have glutamate at this position; in most other transporters, this residue is aspartate. To test functional differences between glutamate and aspartate at this position, we constructed the E493D mutant in rSERT and expressed it in Xenopus oocytes. The E493D mutant displayed $\mathrm{H}^{+}$leakage current (Fig. $8 A$ ) and transient current (Fig. $8 B$ ); the amplitudes of these two currents were similar to those of WT, suggesting a similar expression level. However, to our surprise, the E493D mutant displayed a much smaller transport-associated current of $<5 \mathrm{nA}$ at $\mathrm{pH} 7.4$ (Fig. 8A) compared with the current of 10-20 nA normally seen with WT rSERT. Furthermore, the transport-associated current was no longer potentiated by acidic $\mathrm{pH}$. 5-HT may even slightly inhibit the $\mathrm{H}^{+}$leakage current (Fig. 8A). To test whether the E493D mutation also affects the charge per 5-HT ratio, we measured $\left[{ }^{3} \mathrm{H}\right] 5$-HT uptake under voltage-clamp conditions. As shown in Figure $8 C$, the charge per 5-HT ratio decreased by $\sim 50 \%$ in E493D (8.1 \pm 2.7 for E493D vs $17.2 \pm 4.8$ for WT; $n=$ 3 ), indicating that there is less uncoupled charge movement per 5-HT molecule transported.

\section{DISCUSSION}

The mutations near position 490 described here produce specific and subtle functional anomalies, encouraging us to assume that the overall structure of SERT remains unchanged. The mutations change the transport-associated current, its dependence on $\mathrm{pH}$, and the number of charges transported per 5-HT. But these mutations do not change the dose-response relation for $5-\mathrm{HT}$, the leakage current, or the voltage-dependent transient current. Although WT rSERT and WT hSERT differ in the binding of tricyclic antidepressants and of D-amphetamine, these differences are governed by residues downstream of position 532 rather than by positions 490 and 493 studied here (Barker et al., 1994).

\section{The gate-lumen-gate model}

We interpret our experiments in terms of a model for neurotransmitter transporter function in which the transport protein contains a channel-like lumen flanked by intracellular and extracellular gates (Fig. 9). These gates open and close individually and sequentially in response to the binding of transported substrates, with resulting changes in compartmentalization that form the essence of coupled transport (Lester et al., 1994, 1996). Although other conceptual models have been advanced (Su et al., 1996), the gate-lumen-gate scheme is useful if the external gate, the lumen, and the internal gate can be localized at the level of primary amino acid sequence (and ultimately at the atomic scale in three dimensions).

We want to state that the scheme of Figure 9 has some heuristic value but that it omits some relevant data on the order and 


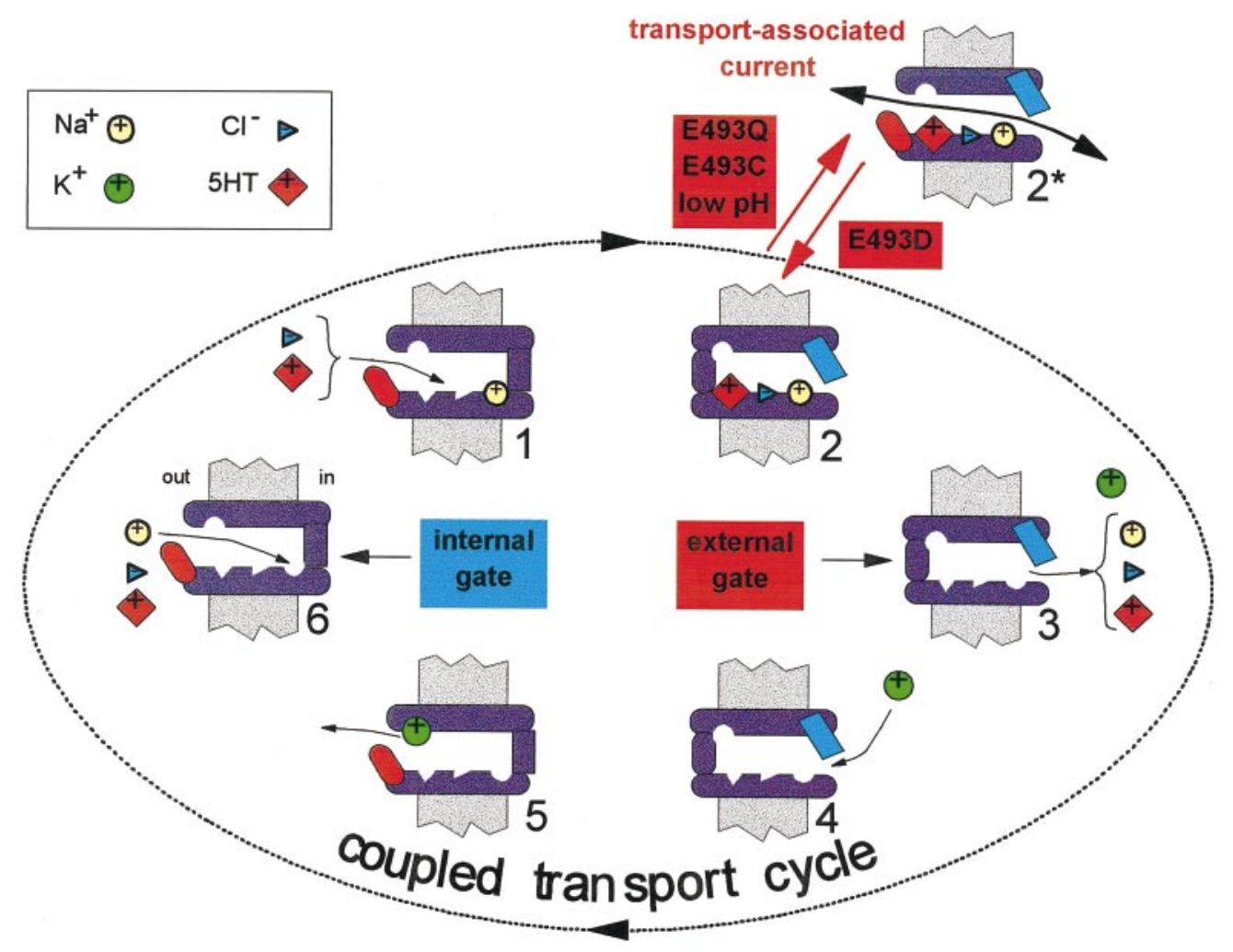

Figure 9. Schematic diagram of the transport cycle for SERT. Six numbered states are enclosed within an oval; arrowheads on the oval denote the direction of normal progression through the transport cycle. Fully coupled stoichiometry applies within the oval; one 5-HT molecule is transported into the cell along with a single $\mathrm{Na}^{+}$ion and a single $\mathrm{Cl}^{-}$ion (states $6,1,2$, and 3). A single $\mathrm{K}^{+}$ion leaves (states 5 and 6). The external gate is labeled in red when open; the internal gate is labeled in blue when open. When 5- $\mathrm{HT}, \mathrm{Na}^{+}$, and $\mathrm{Cl}^{-}$bind within the lumen, the external gate closes, and the internal gate opens (state $1 \rightarrow$ state 2 ). This changes the compartmentalization of the three substrates, which accounts for their coupled flux. When $\mathrm{K}^{+}$then binds within the lumen, the internal gate closes, and the external gate opens, accounting for the obligatory role of $\mathrm{K}^{+}$(state $4 \rightarrow$ state 5 ). Outside the oval is the state $\left(2^{*}\right)$ corresponding to the uncoupled transport-associated current. We hypothesize that this uncoupled transport-associated current represents a violation of the alternating-access rules; both gates are open simultaneously. Retaining the convention from the ion channel literature, we describe this conducting state by an *. The red arrows indicate probabilities, not formal rate constants; E493Q, E493C, and low pH increase the probability of state $2^{*}$, whereas E493D decreases this probability.

cooperativity of substrate binding (Rudnick and Clark, 1993) and that it has no known relation to structural features on the atomic scale. The illustration shows six numbered states enclosed within an oval; arrowheads on the oval denote the direction of normal progression through the transport cycle. Within the oval, the accepted substrate stoichiometry prevails; one 5-HT molecule is transported into the cell along with a single $\mathrm{Na}^{+}$ion and a single $\mathrm{Cl}^{-}$ion. A single $\mathrm{K}^{+}$ion leaves. When 5-HT, $\mathrm{Na}^{+}$, and $\mathrm{Cl}^{-}$bind within the lumen, the external gate closes, and the internal gate opens. This changes the compartmentalization of the three substrates, which accounts for their coupled flux. When $\mathrm{K}^{+}$then binds within the lumen, the internal gate closes, and the external gate opens, accounting for the obligatory role of $\mathrm{K}^{+}$.

Outside the oval is the uncoupled transport-associated current that we have studied and manipulated in these experiments. We hypothesize that this uncoupled current represents a violation of the alternating-access rules; both gates are open simultaneously (Lester et al., 1996; Sonders and Amara, 1996). An analogous state $1^{*}$ can be suggested for the leakage current; indeed, we have found mutations affecting that current as well, and these will be reported separately. We do not believe that these currents are obligatory steps in the transport cycle (Lin et al., 1996, but see Galli et al., 1996); however, we have suggested that the various currents represent important constraints on the mechanism of transport (Lester et al., 1996).

\section{The external gate may be near position $\mathbf{4 9 0}$}

Position 490 and nearby residues are good candidates for partial involvement in external gating. We know that the region is extracellular, because the present data show that the 493C mutant is accessible to extracellular cysteine reagents. Mutations in this region change the $\mathrm{pH}$ sensitivity of the transport-associated current. The mutation could either change the conductance or affect the gating process (changing the open probability). However, the first possibility is unlikely because (1) E493 is located in an extracellular loop and is thus unlikely to line the permeation pathway and (2) the amplitude of the leakage current is not affected by this mutation. We thus favor the second possibility, that either the E493C mutation, the E493Q mutation, or low pH increase the open probability of the external gate. We schematize this influence (Fig. 9) by the red arrows connecting state 2 with the conducting state $2^{*}$, in which the external gate is abnormally open, whereas the internal gate is normally open.

Although the amino acid residue difference at position 490 is responsible for the different $\mathrm{pH}$ sensitivities of hSERT and rSERT, E493 in rSERT might be the actual residue that is protonated to cause increased transport-associated current. The positively charged lysine at 490 in hSERT would be one turn away from E493 in an $\alpha$-helix. This lysine might prevent a proton from gaining access to E493 and thus abolish the low-pH potentiation. 
More specifically, the lysine might form a salt bridge with E493, stabilizing E493 in the charged form and thus stabilizing hSERT in a state equivalent to that of rSERT at higher $\mathrm{pH}$. The requirement for glutamate is very strict; even the conservative substitution of aspartate abolishes the potentiation. Striking differences in coupling and $\mathrm{pH}$ dependence are caused by glutamate versus aspartate, in some cases because of salt bridges with lysine, in two other ion-coupled transporters: the Escherichia coli lactose permease (Sahin-Toth and Kaback, 1993) and the vesicular monoamine transporter (Merickel et al., 1997).

Several other neurotransmitter transporters tested to date have not revealed the low-pH potentiation of the transport-associated current (Cao et al., 1997). There are two possible bases for this unique behavior. (1) SERT is unusual because it is not electrogenic. For the GABA transporter GAT1, the transport-associated current is tightly coupled to the transport cycle; that is, the transport-associated current is explained by the stoichiometry of the events that occur within the oval of Figure 9 (Kavanaugh et al., 1992). The transport-associated current for SERT may arise instead from fortuitous operation of the gates, as described above. (2) Most other neurotransmitter transporters have aspartate at the position that aligns with 493 (Fig. 1C). Our results show that this residue abolishes the low-pH potentiation of rSERT and also reduces the transport-associated current at neutral $\mathrm{pH}$ (Fig. 8). An interesting question is whether the increased transportassociated current, governed in part by glutamate at position 493, has a physiological role either via the resulting depolarization or via the accumulation of internal $\mathrm{Na}^{+}$. Related roles have been suggested for the $\mathrm{Cl}^{-}$conductance associated with glutamate transporters (Sonders and Amara, 1996).

\section{Progress in other studies}

The S545A mutation in TM11 (marked as number 4 in Fig. 1A) decreases the $\mathrm{Na}^{+}$specificity of transport (Sur et al., 1997). In external NMDG Ringer's solution, S545A shows no detectable transport. Compared with WT, S545A displays an increased $\mathrm{EC}_{50}$ for 5-HT and a reduced cooperativity for $\mathrm{Na}^{+}$binding (Sur et al., 1997). The results with S545A suggested to Sur et al. (1997) that S545 is a component of the external gate and participates in initial $\mathrm{Na}^{+}$binding. The gates are likely to comprise several portions of the transporter, in addition to the region suggested by our own studies.

There has also been progress in localizing the lumen. A mutation at G177 in TM3 (one of the mutations noted by number 2 in Fig. 1 $A$ ) increases single-channel conductance (Lin et al., 1996). Cysteine residues introduced into TM3 are accessible to alkylating reagents at several positions, and the periodicity suggests an $\alpha$-helical structure (also noted as number 2 in Fig. $1 A$ ) (Chen et al., 1997b), suggesting that TM3 may comprise part of the lumen. Results to date therefore suggest that the gate-lumen-gate scheme provides one possible theoretical framework, and perhaps a preliminary structural framework, for explaining SERT function.

\section{REFERENCES}

Amara SG, Kuhar M (1993) Neurotransmitter transporters: recent progress. Annu Rev Neurosci 16:73-93.

Barker EL, Kimmel HL, Blakely RD (1994) Chimeric human and rat serotonin transporters reveal domains involved in recognition of transporter ligands. Mol Pharmacol 46:799-807.
Blakely RD, Berson HE, Fremeau RT, Jr., Caron MG, Peek MM, Prince HK, Bradley CC (1991) Cloning and expression of a functional serotonin transporter from rat brain. Nature 354:66-70.

Buck KJ, Amara SG (1994) Chimeric dopamine-norepinephrine transporters delineate structural domains influencing selectivity for catecholamines and 1-methyl-4-phenylpyridinium. Proc Natl Acad Sci USA 91:12584-12588.

Buck KJ, Amara SG (1995) Structural domains of catecholamine transporter chimeras involved in selective inhibition by antidepressants and psychomotor stimulants. Mol Pharmacol 48:1030-1037.

Cao Y, Mager S, Lester HA (1997) $\mathrm{H}^{+}$permeation and $\mathrm{pH}$ regulation at a mammalian serotonin transporter. J Neurosci 17:2257-2266.

Chen JG, Liu-Chen S, Rudnick G (1997a) External cysteine residues in the serotonin transporter. Biochemistry 36:1479-1486.

Chen JG, Sachpatzidis A, Rudnick G (1997b) The third transmembrane domain of the serotonin transporter contains residues associated with substrate and cocaine binding. J Biol Chem 272:28321-28327.

Collier DA, Stober G, Li T, Heils A, Catalano M, Di Bella D, Arranz MJ, Murray RM, Vallada HP, Bengel D, Muller CR, Roberts GW, Smeraldi E, Kirov G, Sham P, Lesch KP (1996) A novel functional polymorphism within the promoter of the serotonin transporter gene: possible role in susceptibility to affective disorders. Mol Psychiatry 1:453-460.

Corey JL, Quick M, Davidson N, Lester HA, Guastella J (1994) A cocaine-sensitive Drosophila serotonin transporter: cloning, expression and electrophysiological characterization. Proc Natl Acad Sci USA 90:1188-1192.

Demchyshyn LL, Pristupa ZB, Sugamori KS, Barker EL, Blakely RD, Wolfgang WJ, Forte MA, Niznik HB (1994) Cloning, expression, and localization of a chloride-facilitated, cocaine-sensitive serotonin transporter from Drosophila melanogaster. Proc Natl Acad Sci USA 91:5158-5162.

Frohman MA, Dush MK, Martin GR (1988) Rapid production of fulllength cDNAs from rare transcripts: amplification using a single genespecific oligonucleotide primer. Proc Natl Acad Sci USA 85:8998-9002.

Galli A, Blakely RD, DeFelice LJ (1996) Norepinephrine transporters have channel modes of conduction. Proc Natl Acad Sci USA 93:86718676.

Galli A, Petersen CI, deBlaquiere M, Blakely RD, DeFelice LJ (1997) Drosophila serotonin transporters have voltage-dependent uptake coupled to a serotonin-gated ion channel. J Neurosci 17:3401-3411.

Giros B, Wang YM, Suter S, McLeskey SB, Pifl C, Caron MG (1994) Delineation of discrete domains for substrate, cocaine, and tricyclic antidepressant interactions using chimeric dopamine-norepinephrine transporters. J Biol Chem 269:15985-15988.

Higuchi R (1990) Recombinant PCR. In: PCR protocols: a guide to methods and applications (Innis MA, Gelfand DH, Sninsky JJ, White TJ, eds). New York: Academic.

Hoffman BJ, Mezey E, Brownstein MJ (1991) Cloning of a serotonin transporter affected by antidepressants. Science 254:579-580.

Kavanaugh MP, Arriza JL, North RA, Amara SG (1992) Electrogenic uptake of $\gamma$-aminobutyric-acid by a cloned transporter expressed in Xenopus oocytes. J Biol Chem 267:22007-22009.

Lesch KP, Wolozin BL, Murphy DL, Riederer P (1993) Primary structure of the human platelet serotonin uptake site: identity with the brain serotonin transporter. J Neurochem 60:2319-2322.

Lesch KP, Bengel D, Heils A, Sabol SZ, Greenberg BD, Petri S, Benjamin J, Muller CR, Hamer DH, Murphy DL (1996) Association of anxiety-related traits with a polymorphism in the serotonin transporter gene regulatory region. Science 274:1527-1531.

Lester HA, Mager S, Quick MW, Corey JL (1994) Permeation properties of neurotransmitter transporters. Annu Rev Pharmacol Toxicol 34:219-249.

Lester HA, Cao Y, Mager S (1996) Listening to neurotransmitter transporters. Neuron 17:807-810.

Lin F, Lester HA, Mager S (1996) Single-channel currents produced by the serotonin transporter, and analysis of a mutation affecting ion permeation. Biophys J 71:3126-3135.

Mager S, Min C, Henry DJ, Chavkin C, Hoffman BJ, Davidson N, Lester HA (1994) Conducting states of a mammalian serotonin transporter. Neuron 12:845-859.

Merickel A, Kaback HR, Edwards RH (1997) Charged residues in transmembrane domains II and XI of a vesicular monoamine transporter form a charge pair that promotes high affinity substrate recognition. J Biol Chem 272:5403-5408.

Nowak MW, Gallivan JP, Silverman SK, Labarca CG, Dougherty DA, 
Lester HA (1998) In vivo incorporation of unnatural amino acids into ion channels in a Xenopus oocyte expression system. Methods Enzymol, in press.

Quick MW, Lester HA (1994) Methods for expression of excitability proteins in Xenopus oocytes. In: Ion channels of excitable cells (Narahashi T, ed), pp 261-279. San Diego: Academic.

Ramamoorthy S, Bauman AL, Moore KR, Han H, Yang-Feng T, Chang AS, Ganapathy V, Blakely RD (1993) Antidepressant- and cocainesensitive human serotonin transporter: molecular cloning, expression, and chromosomal localization. Proc Natl Acad Sci USA 90:2542-2546.

Rudnick G, Clark J (1993) From synapse to vesicle: the reuptake and storage of biogenic amine neurotransmitters. Biochim Biophys Acta 1144:249-263.
Sahin-Toth M, Kaback HR (1993) Properties of interacting aspartic acid and lysine residues in the lactose permease of Escherichia coli. Biochemistry 32:10027-10035.

Sonders MK, Amara SG (1996) Channels in transporters. Curr Opin Neurobiol 6:294-302.

Su A, Mager S, Mayo SL, Lester HA (1996) A multi-substrate single-file model for ion-coupled transporters. Biophys J 70:762-777.

Sur C, Betz H, Schloss P (1997) A single serine residue controls the cation dependence of substrate transport by the rat serotonin transporter. Proc Natl Acad Sci USA 94:7639-7644.

Usdin TB, Mezey E, Chen C, Brownstein MJ, Hoffman BJ (1991) Cloning of the cocaine-sensitive bovine dopamine transporter. Proc Natl Acad Sci USA 88:11168-11171. 